$\underline{\text { Preprint typeset in JHEP style. - HYPER VERSION }}$

SISSA $1 / 99 / \mathrm{EP}$

January 1999

\title{
D-particles in the space-time of the shock wave
}

\author{
Ciprian Acatrinei, Marco Fabbrichesi and Roberto lengo \\ INFN, Sezione di Trieste and \\ Scuola Internazionale Superiore di Studi Avanzati (SISSA) \\ via Beirut 4, I-34013 Trieste, Italy.
}

\begin{abstract}
We study the interaction of two D-particles in the space-time of the shock wave. We first write the amplitude in string theory and find that, at large distances from the shock-wave source, the $O\left(v^{4}\right)$ term in the relative velocity $v$ is an $\alpha^{\prime}$-independent function of the interbrane separation $b$. The amplitude is therefore that of supergravity - for large $b$, only closed-string massless modes contribute. We then show how the same result is obtained in the matrix model (at small $b$ ) by setting up the formulation of the dimensionally reduced super Yang-Mills theory in the curved background of the shock wave.
\end{abstract}

KEYwords: M(atrix) Theories, D-branes, Superstrings and Heterotic Strings. 


\section{Contents}

1. Motivations and Summary 1

2. String theory 3

2.1. String center-of-mass modes 5

2.2. Oscillator modes 6

2.3. The final amplitude 8

3. The matrix model in the shock wave 9

3.1. Kinematics 10

3.2. The quadratic action 12

3.3. The scattering amplitude 14

3.4. Comparison with the string result 15

A. Notation and useful formulas

A.1. String propagators 16

A.2. Shock-wave metric 17

\section{Motivations and Summary}

D-particles (D0-branes) are the simplest example of Dirichlet branes [1]. Their dynamics has been studied in order to understand their role in string theory and to compare their behavior with that of gravitons having momentum in a compactified direction in the dimensionally reduced $d=11$ supergravity theory (that is, the long-distance limit of M theory). This comparison is important in identifying which dynamical configurations are susceptible of a description in terms of light open-string degrees of freedom [2, [3], thus providing the effective "low energy" theory of D-branes that is at the basis of the matrix model [4]. This issue has been analyzed both in the case of two-body interactions [5] and in the more arduous case of three bodies [6, 7] (see also [8] for a related temporary controversy).

A better understanding of the physics of D-brane in non-flat space-time is also desirable because it is a subject relevant to many open questions in the interplay between string theory and gravitation. At the moment, very little is known about the matrix model in curved spaces [9]. Recent analyses discuss the case of the Schwarzschild metric [10]. The present paper is a first enquire in this direction. 
One of the simplest examples of dynamics in curved space is the motion of two D-particles in the so-called shock-wave metric, that is, the gravitational field generated by a massless particle. Since there are two particles in addition to the massless source of gravity, this is also a case of a three-body interaction, which is of special interest because of the inherent peculiarities of the three-body dynamics. Moreover, the shockwave metric itself is particularly important in the study of quantum gravity, see [11, 12, 13, 14, 15] and, recently, [16].

In order to pursue our program, we approach the problem from two different points of view.

First, we perform an analysis in terms of closed-string theory. Since the simplest massless particle in this theory is the graviton, we take it to be the source for the shock-wave metric.

We write the amplitude for the scattering of two D-particles and two gravitons describing the incoming and outgoing massless source. The process in which we are interested emerges as a pinching limit of the full amplitude, where the two graviton vertex operators collide in the world-sheet, implementing within the string algorithm the emission of the long-range gravitational field (the shock-wave) from the incomingoutgoing source. The full amplitude in this pinching limit describes the interaction of two D-branes with themselves and with the shock-wave.

In this computational framework, the D-particles are special boundary states at the ends of a cylindrical world-sheet, and the string amplitude is obtained by computing the correlator of the two vertices on the cylinder (we consider the ten dimensional uncompactified space-time). The D-particles are treated in the usual eikonal approximation, however the computation of the amplitude is not quite conventional because of the peculiarities of the three-body kinematics. After summing over the spin-structures we look at the expansion in the relative velocity $v$ of the D-particles.

The first result of our work is that, up to the fourth order in $v$, the leading singularities in the momentum transfer from the source-gravitons are a function of the interbrane distance $b$ that is the same for arbitrary $b$ and therefore independent from $\alpha^{\prime}$, see eq. (2.36). This result generalizes well-known properties of brane interaction to the case of a non-trivial background. The leading singularities in the momentum transfer correspond to the leading powers of the transverse distance $r_{\top}$ from the shock-wave source. In particular, the fourth order term in $v$ gets contribution from the leading behavior of the shock-wave gravitational field, that is $O\left(r_{\top}{ }^{-6}\right)$, whereas the second order term is sub-leading and $O\left(r_{T^{-8}}{ }^{-8}\right.$. We will focus on the fourth order term.

Of course, the long interbrane-distance regime is dominated by the massless modes of the closed string and is therefore the supergravity (and M theory) result.

On the other hand, we can consider the same process in the short interbrane-distance regime where we expect the theory to be described by the dimensionally reduced $d=10$ super Yang-Mills theory corresponding to the exchange of massless open-string states. The gauge group is $S U(2)$ because we want to describe the interaction of two D- 
particles. The novelty is here that the super Yang-Mills theory must be written in a gravitational background. This frames the problem in the language of the matrix model, for which the D-particles are the fundamental degrees of freedom, in the rather unconventional case of a curved background. The final amplitude describes the motion of two D-particles in the space-time of the shock wave.

We show how, by performing the small $v$ and large $r_{\top}$ expansion of the matrix-model result, the amplitude of the closed-string computation is reproduced, see eq. (3.51).

\section{String theory}

Consider two D-particles located at $\vec{Y}_{1}^{\perp}$ and $\vec{Y}_{2}^{\perp}$ and moving with velocities $\vec{v}_{1}$ and $\vec{v}_{2}$, respectively, where $\vec{Y}_{(i)}^{\perp} \cdot \vec{v}_{(i)}=0$. We consider the frame where $\vec{v}_{1}+\vec{v}_{2}=\vec{Y}_{1}^{\perp}+\vec{Y}_{2}^{\perp}=0$. We call $\vec{v}=\vec{v}_{1}-\vec{v}_{2}$ and $\vec{b}=\vec{Y}_{1}^{\perp}-\vec{Y}_{2}^{\perp}$, where by definition $\vec{b} \cdot \vec{v}=0$. Their interaction with an external gravitation field - generated by the source graviton moving along the direction $z$ at a transverse distance $\vec{r}_{\top}$ (by definition $\vec{r}_{\top}$ is orthogonal to $z$ ) from the center of mass of the two D-particles - is dominated by a term proportional to $1 / q^{2}$, where $q$ is the momentum transfer by the gravitation field to the system of the two D-particles. The momentum $\vec{q}$ can be separated into a part $q_{z}$ along the direction of motion of the external graviton (i.e., the shock-wave direction), and the remaining orthogonal directions, $\vec{q}_{\top}$. In the eikonal approximation, $q_{0}=q_{z}$ that implies $q^{2}=q_{\top}^{2}$.

In the frame chosen, the motion of the center of mass of the particles is factorized out. The effect of the external source graviton on it is taken into account by disconnected diagrams - which we do not consider - in which the source couples independently first to one of the two D-particles and then to the other. Here we focus instead on the relative motion of the two D-particles as they interact with the gravitational field.

We write the amplitude in $d=10$ as

$$
a\left(r_{T}, b, v\right)=\int \frac{\mathrm{d} q_{z} \mathrm{~d}^{d-2} \vec{q} \uparrow}{(2 \pi)^{d-1}} e^{i \vec{r} \cdot \vec{q} \top} \int \mathrm{d} \ell \int \mathrm{d}^{2} w \mathrm{~d}^{2} z \mathcal{A}(z, w ; \ell),
$$

where (up to an overall constant)

$$
\mathcal{A}(z, w ; \ell)=\sum_{S}( \pm)_{S}\left\langle v_{2}, Y_{2}\left|e^{-\ell H} V_{k}(z, \bar{z}) V_{p}(w, \bar{w})\right| v_{1}, Y_{1}\right\rangle_{S}
$$

contains the interaction of the two D-particles with the two gravitons, the vertices of which are given by

$$
V_{p}(z, \bar{z})=\varepsilon_{\mu \nu}^{(p)}\left[\partial X^{\mu}(z)+i p \cdot \psi(z) \psi^{\mu}(z)\right]\left[\bar{\partial} X^{\nu}(\bar{z})+i p \cdot \bar{\psi}(\bar{z}) \bar{\psi}^{\nu}(\bar{z})\right] e^{i p \cdot X}
$$

and similarly for the other one at $w$ and carrying momentum $k$. The index $S$ stands for the three even spin structures of the fermionic propagators. The amplitude (2.2), in the case of $\vec{v}=0$, has been considered in [17. 
For later use, we define the two vectors $q$ and $P$ to be

$$
q_{0}=k_{0}+p_{0}, \quad \vec{q}=\vec{k}+\vec{p} \quad \text { and } \quad P_{0}=k_{0}-p_{0}, \quad \vec{P}=\vec{k}-\vec{p} .
$$

The D-particle kets or bras are given by a tensor product of center-of-mass and string-oscillator states. The D-particle center-of-mass state in space-time is described by a Fourier transform in terms of the eigenstates of the energy-momentum transfer $Q$.

One has further to integrate over the world-line of the D-particle, which in the eikonal approximation is the straight line $Y^{\mu}(t)$ parameterized by $Y(t)=(t, \vec{R}(t))$ with $\vec{R}=\vec{v} t+\vec{Y}_{\perp}$. We thus get

$$
\left|v, Y_{\perp}\right\rangle=\int \mathrm{d} t \int \frac{\mathrm{d}^{d} Q}{(2 \pi)^{d}} e^{i\left(Q_{0} t-\vec{Q} \cdot \vec{R}(t)\right)}|v, Q\rangle=\int \frac{\mathrm{d}^{d-1} \vec{Q}}{(2 \pi)^{d-1}} e^{i \vec{Q} \cdot \vec{Y}_{\perp}}\left|v, Q_{0}=\vec{v} \cdot \vec{Q}, \vec{Q}\right\rangle
$$

Notice that in the eikonal approximation we have the constraint $Q_{0 i}=\vec{v}_{i} \cdot \vec{Q}_{i} \equiv v_{i} Q_{L}^{i}$. This is similar to what holds for the momentum transfer from the source graviton (whose velocity is equal to 1 along the $z$-direction) namely $q_{0}=q_{z}$.

In eq. (2.2) the Hamiltonian $H$ transports the D-particle at $\vec{Y}_{2}^{\perp}$ and is given by

$$
H=H_{\mathrm{CM}}+H_{\mathrm{OS}}=\frac{1}{2} Q_{2}^{2}+H_{\mathrm{OS}},
$$

where $H_{\mathrm{OS}}$ contains the string oscillators.

Let us also define the normalized correlators

$$
\left\langle V_{k}(z, \bar{z}) V_{p}(w, \bar{w})\right\rangle_{S}=\frac{\left\langle v_{2}, Y_{2}\left|e^{-\ell H} V_{k}(z, \bar{z}) V_{p}(w, \bar{w})\right| v_{1}, Y_{1}\right\rangle_{S}}{\left\langle v_{2}, Y_{2}\left|e^{-\ell H_{\mathrm{OS}}}\right| v_{1}, Y_{1}\right\rangle_{S}},
$$

where

$$
\left\langle v_{2}, Y_{2}\left|e^{-\ell H_{\mathrm{OS}}}\right| v_{1}, Y_{1}\right\rangle_{S}=\frac{\vartheta_{S}(i v / \pi) \vartheta_{S}^{3}(0)}{\vartheta_{1}^{\prime 4}(0)},
$$

and therefore

$$
\mathcal{A}(z, w ; \ell)=\sum_{S}( \pm)_{S}\left\langle V_{k}(z, \bar{z}) V_{p}(w, \bar{w})\right\rangle_{S} \frac{\vartheta_{S}(i v / \pi) \vartheta_{S}^{3}(0)}{\vartheta_{1}^{\prime 4}(0)} .
$$

Since we are not interested in spin effects, we can consider only the term proportional to $\varepsilon^{(p)} \cdot \varepsilon^{(k)}$. The two-graviton correlation function is then

$$
\begin{aligned}
\left\langle V_{k}(z, \bar{z}) V_{p}(w, \bar{w})\right\rangle_{S} & =\varepsilon_{\mu \lambda}^{(p)} \varepsilon_{\nu \rho}^{(k)}\left[\left\langle\partial X^{\mu} \partial X^{\lambda}\right\rangle\left\langle\bar{\partial} X^{\nu} \bar{\partial} X^{\rho}\right\rangle\right. \\
& -\left\langle\psi^{\mu} \psi^{\lambda}\right\rangle_{S}\left\langle\bar{\psi}^{\nu} \bar{\psi}^{\rho}\right\rangle_{S}\langle p \cdot \psi p \cdot \bar{\psi} k \cdot \psi k \cdot \bar{\psi}\rangle_{S} \\
& +\left\langle\partial X^{\mu} \partial X^{\lambda}\right\rangle\left\langle\bar{\psi}^{\nu} \bar{\psi}^{\rho}\right\rangle_{S}\langle p \cdot \bar{\psi} k \cdot \bar{\psi}\rangle_{S} \\
& \left.+\left\langle\bar{\partial} X^{\mu} \bar{\partial} X^{\lambda}\right\rangle\left\langle\psi^{\nu} \psi^{\rho}\right\rangle_{S}\langle p \cdot \psi k \cdot \psi\rangle_{S}\right]\left\langle e^{i k \cdot X(z)} e^{i p \cdot X(w)}\right\rangle .
\end{aligned}
$$


We look for the singularities as we take the limit $z \rightarrow w$ :

$$
\begin{aligned}
\left\langle V_{k}(z, \bar{z}) V_{p}(w, \bar{w})\right\rangle_{S} \rightarrow & \frac{\varepsilon^{(p)} \cdot \varepsilon^{(k)}}{(4 \pi)^{2}}\left\{\frac{1}{|z-w|^{4}}\left(1+O\left(q^{2}\right)\right)\right. \\
& -\frac{1}{|z-w|^{2}}\left[\langle k \cdot \psi k \cdot \bar{\psi}\rangle_{S}\langle p \cdot \psi p \cdot \bar{\psi}\rangle_{S}\right. \\
& \left.\left.+\langle k \cdot \psi p \cdot \bar{\psi}\rangle_{S}\langle k \cdot \bar{\psi} p \cdot \psi\rangle_{S}+O\left(q^{4}\right)\right]\right\}\left\langle e^{i k \cdot X(z)} e^{i p \cdot X(w)}\right\rangle .
\end{aligned}
$$

As we shall see, we can neglect in (2.11) $O\left(q^{2}\right)$ terms in the quartic pole and $O\left(q^{4}\right)$ terms in the quadratic pole.

While the second term, which comes from the fermionic correlators, is already a quadratic pole, the first one, which comes from the bosonic correlators, is quartic and, in order to contribute, it must be multiplied by terms containing a factor $|z-w|^{2}$. In fact, it is the quadratic pole times a factor $|z-w|^{q^{2} /(4 \pi)}$, contained in $\left\langle e^{i k \cdot X} e^{i p \cdot X}\right\rangle$, that gives rise to the singularity $1 / q^{2}$ for which we are looking:

$$
\int \mathrm{d}^{2}(z-w)|z-w|^{-2+q^{2} /(4 \pi)}=\frac{8 \pi^{2}}{q^{2}} .
$$

We split the center-of-mass modes $X_{0}$ from the string-oscillator modes in the correlator:

$$
\left\langle e^{i k \cdot X(z)} e^{i p \cdot X(w)}\right\rangle=\left\langle e^{i k \cdot X_{0}(z)} e^{i p \cdot X_{0}(w)}\right\rangle\left\langle e^{i k \cdot X(z)} e^{i p \cdot X(w)}\right\rangle_{\text {os }},
$$

and analyze their contributions separately.

\subsection{String center-of-mass modes}

Let us first consider the string center-of-mass modes. They are given by

$$
X_{0}^{\mu}(z)=X_{0}^{\mu}-i Q^{\mu} \operatorname{Im} z,
$$

where $\operatorname{Im} z$ plays the role of proper time of closed string propagating from one D-particle to the other one.

Because

$$
\begin{gathered}
\left\langle\vec{Q}_{2}, Q_{2}^{0}\right| e^{i k \cdot X(0)}=\left\langle\vec{Q}_{2}-\vec{k}, Q_{2}^{0}-k_{0}\right| \\
e^{i p \cdot X(0)}\left|\vec{Q}_{1}, Q_{1}^{0}\right\rangle=\left|\vec{Q}_{1}+\vec{p}, Q_{1}^{0}+p_{0}\right\rangle,
\end{gathered}
$$

we have two conservation laws given by the sandwiching of the external states:

$$
\begin{aligned}
\left\langle\vec{Q}_{2}-\vec{k}, Q_{2}^{0}-k_{0} \mid \vec{Q}_{1}+\vec{p}, Q_{1}^{0}+p_{0}\right\rangle= & \delta^{(d-1)}\left(\vec{Q}_{2}-\vec{k}-\vec{Q}_{1}-\vec{p}\right) \\
& \times \delta\left(Q_{2}^{0}-k_{0}-Q_{1}^{0}-p_{0}\right) .
\end{aligned}
$$

Notice that the energy conservation gives $\vec{Q}_{2} \cdot \vec{v}_{2}-\vec{Q}_{1} \cdot \vec{v}_{1}=q_{z}$. 
According to (2.7), for

$$
Z_{\mathrm{CM}} \equiv\left\langle e^{i k \cdot X_{0}(z)} e^{i p \cdot X_{0}(w)}\right\rangle=\left\langle v_{2}, Y_{2}\left|e^{-\ell H_{\mathrm{CM}}} e^{i k \cdot X_{0}(z)} e^{i p \cdot X_{0}(w)}\right| v_{1}, Y_{1}\right\rangle
$$

we have

$$
\begin{aligned}
Z_{\mathrm{CM}}= & \int \frac{\mathrm{d}^{d-1} \vec{Q}_{1}}{(2 \pi)^{D-1}} \int \frac{\mathrm{d}^{d-1} \vec{Q}_{2}}{(2 \pi)^{D-1}} \exp \left[i \vec{Q}_{1} \cdot Y_{\perp}^{1}-i \vec{Q}_{2} \cdot Y_{\perp}^{2}\right] \\
& \times \exp \left\{-\frac{\ell}{2}\left[\vec{Q}_{2}^{2}-\left(\vec{v}_{2} \cdot Q_{2}\right)^{2}\right]+\operatorname{Im} z\left(\vec{k} \cdot \vec{Q}_{2}-k_{0} \vec{v}_{2} \cdot \vec{Q}_{2}\right)\right. \\
& \left.+\operatorname{Im} w\left(\vec{p} \cdot \vec{Q}_{1}-p_{0} \vec{v}_{1} \cdot \vec{Q}_{1}\right)\right\} \delta^{(d)}\left(Q_{2}-Q_{1}-k-p\right) .
\end{aligned}
$$

We replace by means of the $\delta$-functions $\vec{Q}_{2}=\vec{Q}_{1}+\vec{q}$; we set, by neglecting $\vec{q} \cdot \vec{v} / v$, $Q_{L}=-q_{z} / v$ and $\vec{Q}_{1}=\vec{Q}$. Finally, we integrate over the components $\vec{Q}_{\perp}$ orthogonal to $\vec{v}$ and find

$$
\begin{aligned}
Z_{\mathrm{CM}}= & -\frac{1}{v} e^{-i \vec{q} \cdot \vec{b} / 2} \int \frac{\mathrm{d}^{d-2} \vec{Q}_{\perp}}{(2 \pi)^{d-2}} \\
& \times \exp \left[-\frac{\ell}{2} \vec{Q}_{\perp}^{2}+(\operatorname{Im} z \vec{k}+\operatorname{Im} w \vec{p}+i \vec{b}) \cdot \vec{Q}_{\perp}\right] \\
& \times \int \frac{\mathrm{d} q_{z}}{2 \pi} \exp \left[-\frac{\ell}{2}\left[1+(\operatorname{Im} z+\operatorname{Im} w) \frac{v_{z}}{\ell}\right]\left(\frac{q_{z}}{v}\right)^{2}\right],
\end{aligned}
$$

which, after integration in $\vec{Q}_{\perp}$ and $q_{z}$, for small $v_{z}$ and $q_{\top} \rightarrow 0$, gives

$$
Z_{\mathrm{CM}}=\ell^{-(d-1) / 2} e^{(\operatorname{Im} z \vec{k}+\operatorname{Im} w \vec{p}+i \vec{b})^{2} / 2 \ell}\left[1-\frac{v_{z}}{2 \ell}(\operatorname{Im} z+\operatorname{Im} w)\right],
$$

where we have dropped overall factors of $2 \pi$.

We retain a term proportional to $|z-w|^{2}$ from the expansion of the exponential in (2.20), and finally obtain

$$
\begin{aligned}
Z_{\mathrm{CM}}= & -\ell^{-(d-1) / 2} e^{-\vec{b}^{2} / 2 \ell}\left\{1-\frac{v_{z}}{2 \ell}(\operatorname{Im} z+\operatorname{Im} w)\right. \\
& \left.+|z-w|^{2}\left[\frac{k_{0}^{2}}{4 \ell}-\frac{1}{16 \ell^{2}}(\vec{P} \cdot \vec{b})^{2}\right]\right\} .
\end{aligned}
$$

\subsection{Oscillator modes}

The oscillator part is given by the expectation value of the exponential factors

$$
\begin{aligned}
\left\langle e^{i k \cdot X(z)} e^{i p \cdot X(w)}\right\rangle_{\mathrm{OS}} & =e^{\left.-\langle[k \cdot X(z)+p \cdot X(w)])^{2}\right\rangle_{\mathrm{OS}} / 2} \\
& =\left|\frac{\vartheta_{1}(z-w)}{\vartheta_{1}(z-\bar{w})}\right|^{q^{2} / 4 \pi}\left|\frac{\vartheta_{1}^{2}(z-\bar{w})}{\vartheta_{1}(z-\bar{z}) \vartheta_{1}(w-\bar{w})}\right|^{k_{0}^{2} / 4 \pi},
\end{aligned}
$$


which in the $z \rightarrow w$ limit gives

$$
\left\langle e^{i k \cdot X(z)} e^{i p \cdot X(w)}\right\rangle_{\mathrm{OS}} \longrightarrow\left[1+|z-w|^{2} \frac{k_{0}^{2}}{2 \pi} \partial_{w}^{2} \ln \vartheta_{1}(w-\bar{w})\right]\left|\frac{\vartheta_{1}(z-w)}{\vartheta_{1}(z-\bar{w})}\right|^{q^{2} / 4 \pi}
$$

where we have kept terms up to $|z-w|^{2}$ as required.

The fermionic correlator can be written (up to $O\left(q^{2}\right)$ ) as

$$
\begin{aligned}
\langle k \cdot \psi k \cdot \bar{\psi}\rangle_{S}\langle p \cdot \psi p \cdot \bar{\psi}\rangle_{S}+ & \langle k \cdot \psi p \cdot \bar{\psi}\rangle_{S}\langle k \cdot \bar{\psi} p \cdot \psi\rangle_{S}= \\
& \left(\frac{1}{4 \pi}\right)^{2}\left\{k_{0}^{2} \frac{(\vec{q} \uparrow \cdot \vec{v})^{2}}{v^{2}}\left[Q_{S}^{2}-R_{S}^{2}-P_{S}^{2}\right]+q^{2} k_{0}^{2}\left[Q_{S} P_{S}+P_{S}^{2}\right]\right. \\
- & \left.q^{2} \frac{(\vec{P} \cdot \vec{v})^{2}}{4 v^{2}}\left[P_{S}^{2}-Q_{S} P_{S}\right]+k_{0} q^{2} \frac{\vec{P} \cdot \vec{v}}{v} R_{S} P_{S}\right\},
\end{aligned}
$$

where the spin-structure dependent functions $Q_{S}, R_{S}$ and $P_{S}$ are given in the appendix.

We perform now the integration

$$
\int \mathrm{d}^{2} z \int \mathrm{d}^{2} w=\int \mathrm{d}^{2}(z-w) \int \mathrm{d} \operatorname{Re} w \int \mathrm{d} \operatorname{Im} w .
$$

Every term in (2.24) is $O\left(q^{2}\right)$. Unless we find an additional singularity coming from the integration over $\operatorname{Im} w$, the $q^{2}$ in front of all terms will cancel the $1 / q^{2}$ coming from the integration over $(z-w)$ and we would be left without the $1 / q^{2}$ pole in which we are interested. We therefore look for terms behaving like $(w-\bar{w})^{-1-q^{2} / 4 \pi}$. After summing over the spin structures, a factor of this form could arise from terms in (2.24) proportional to $Q_{S}^{2}, R_{S}^{2}, Q_{S} P_{S}$ and $R_{S} P_{S}$. The function $P_{S}^{2}$ does not contain the required term. The contributions coming from the terms proportional to $Q_{S}^{2}$ and $R_{S}^{2}$ are equal and therefore cancel. Therefore, there are only two terms in (2.24) that can give rise to the required power of $v$ and $1 / \vec{q}_{\top}^{2}$ :

$$
\begin{aligned}
\langle k \cdot \psi k \cdot \bar{\psi}\rangle_{S}\langle p \cdot \psi p \cdot \bar{\psi}\rangle_{S}+ & \langle k \cdot \psi p \cdot \bar{\psi}\rangle_{S}\langle k \cdot \bar{\psi} p \cdot \psi\rangle_{S} \\
& \longrightarrow\left(\frac{1}{4 \pi}\right)^{2}\left\{k_{0} q^{2} \frac{\vec{P} \cdot \vec{v}}{v} R_{S} P_{S}+q^{2}\left[k_{0}^{2}+\frac{(\vec{P} \cdot \vec{v})^{2}}{4 v^{2}}\right] Q_{S} P_{S}\right\}
\end{aligned}
$$

The sum over the spin structures yields, for $v \rightarrow 0$,

$$
\begin{aligned}
\sum_{S}( \pm)_{S} R_{S} P_{S} \frac{\vartheta_{S}(-i v / \pi) \vartheta_{S}^{3}(0)}{\vartheta_{1}^{\prime}(0)}= & \frac{1}{2} \sum_{S}( \pm)_{S} \frac{\vartheta_{S}(w-\bar{w}) \vartheta_{S}^{2}(0)}{\vartheta_{1}^{2}(w-\bar{w}) \vartheta_{1}^{\prime 2}(0)} \\
& \times\left[e^{v} \vartheta_{S}(w-\bar{w}-i v / \pi)-e^{-v} \vartheta_{S}(w-\bar{w}+i v / \pi)\right] \\
& \longrightarrow \frac{i}{\pi} \frac{v^{3}}{(2 \pi)^{2}} \frac{\vartheta_{1}^{\prime}(w-\bar{w})}{\vartheta_{1}(w-\bar{w})},
\end{aligned}
$$

and

$$
\begin{aligned}
\sum_{S}( \pm)_{S} Q_{S} P_{S} \frac{\vartheta_{S}(-i v / \pi) \vartheta_{S}^{3}(0)}{\vartheta_{1}^{\prime 4}(0)}= & \frac{1}{2} \sum_{S}( \pm)_{S} \frac{\vartheta_{S}(w-\bar{w}) \vartheta_{S}^{2}(0)}{\vartheta_{1}^{2}(w-\bar{w}) \vartheta_{1}^{\prime 2}(0)} \\
& \times\left[e^{v} \vartheta_{S}(w-\bar{w}-i v / \pi)+e^{-v} \vartheta_{S}(w-\bar{w}+i v / \pi)\right] \\
& \longrightarrow 2\left(\frac{i v}{2 \pi}\right)^{4} \frac{\vartheta_{1}^{\prime \prime}(w-\bar{w})}{\vartheta_{1}(w-\bar{w})}
\end{aligned}
$$


after dropping a total derivative, which does not contribute because the contributions at the integration limits cancel against each other.

\subsection{The final amplitude}

We can now collect all the relevant terms by putting in evidence the integral over $z$ around the quadratic pole and writing everything else for $z=w$. The integrand does not depend on Re $w$ and its integration gives a factor 1 . We thus obtain

$$
\begin{aligned}
a\left(r_{\top}, b, v\right) \simeq & -\int \frac{\mathrm{d}^{d-2} \overrightarrow{q_{\top}}}{(2 \pi)^{d-1}} e^{i \vec{r} \cdot \vec{q} \top} e^{-\vec{b}^{2} / 2 \ell} \int \mathrm{d} \ell \ell^{-(d-1) / 2} \\
& \times \int \mathrm{d}^{2}(z-w)|z-w|^{-2+q^{2} /(4 \pi)} \int_{0}^{\ell} \mathrm{d} \operatorname{Im} w\left[F_{2}(w, \bar{w})+F_{4}(w, \bar{w})\right] .
\end{aligned}
$$

In eq. (2.29), $F_{4}(w, \bar{w})$ comes from the bosonic correlator, the term proportional to $|z-w|^{-4}$ in (2.10), times the terms proportional to $|z-w|^{2}$ in (2.21) and (2.23) which compensate for the quartic pole, and is given by

$$
F_{4}(w, \bar{w})=\left(\frac{i v}{2 \pi}\right)^{4}\left[\frac{k_{0}^{2}}{2 \pi} \partial_{w}^{2} \ln \vartheta_{1}(w-\bar{w})+\frac{k_{0}^{2}}{4 \ell}-\frac{1}{16} \frac{\vec{b} \cdot \vec{P}}{\ell^{2}}\right],
$$

where the factor in front comes from the expansion in $v$ of the $\vartheta_{1}(i v / 2 \pi)$ obtained after summing over the spin structures (see formulas in the appendix).

The term $F_{2}(w, \bar{w})$ in eq. (2.29) originates from the fermionic correlators, the term proportional to $|z-w|^{-2}$ in (2.10), and it has two terms, one that originates from (2.28) and one from the product of the fermionic correlator (2.27) and the term proportional to $v_{z}$ in $(2.20)$ :

$$
\begin{aligned}
F_{2}(w, \bar{w})= & -2 \frac{k_{0}^{2} q^{2}}{(4 \pi)^{2}}\left[\left(1+\frac{v_{z}^{2}}{v^{2}}\right)\left(\frac{i v}{2 \pi}\right)^{4} \frac{\vartheta_{1}^{\prime \prime}(w-\bar{w})}{\vartheta_{1}(w-\bar{w})}\right. \\
& \left.-\frac{v_{z}}{v}\left(\frac{i v^{3}}{4 \pi^{3}}\right) \frac{\vartheta_{1}^{\prime}(w-\bar{w})}{\vartheta_{1}(w-\bar{w})}\left(\frac{v_{z}}{\ell}\right) \operatorname{Im} w\right]\left[\vartheta_{1}(w-\bar{w})\right]^{-q^{2} /(4 \pi)}
\end{aligned}
$$

We use

$$
\vartheta_{1}^{\prime}(w-\bar{w})\left[\vartheta_{1}(w-\bar{w})\right]^{-q^{2} /(4 \pi)-1}=\frac{2 i \pi}{q^{2}} \frac{\partial}{\partial \operatorname{Im} w}\left[\vartheta_{1}(w-\bar{w})\right]^{-q^{2} /(4 \pi)},
$$

after which, the integrals over $\operatorname{Im} w$ give (at the leading order in $q^{2} \rightarrow 0$ )

$$
\begin{gathered}
\int_{0}^{\ell} \partial_{w}^{2} \ln \vartheta_{1}(w-\bar{w}) \mathrm{d} \operatorname{Im} w=-\pi \\
\int_{0}^{\ell} \operatorname{Im} w \frac{\partial}{\partial \operatorname{Im} w}\left[\vartheta_{1}(w-\bar{w})\right]^{-q^{2} /(4 \pi)} \mathrm{d} \operatorname{Im} w=-\ell
\end{gathered}
$$

and

$$
\int_{0}^{\ell} \frac{\vartheta_{1}^{\prime \prime}(w-\bar{w})}{\left[\vartheta_{1}(w-\bar{w})\right]^{1+q^{2} /(4 \pi)}} d \operatorname{Im} w=-\frac{8 \pi^{2}}{q^{2}}
$$


respectively. In eq. (2.35), only the upper limit of integration contributes and we have used the relation $\vartheta_{1}(2 i \ell-y)=e^{2 \pi \ell+2 \pi i y} \vartheta_{1}(-y)$.

At this point, the integration over $\mathrm{d}^{2}(z-w)$ in $(2.29)$ gives the desired quadratic pole and we are left with the final result

$$
\begin{aligned}
a\left(r_{\top}, b, v\right) \simeq & k_{0}^{2} \int \frac{\mathrm{d}^{d-2} \vec{q}_{\top}}{(2 \pi)^{d-2}} \frac{1}{\vec{q}_{\top}^{2}} e^{i \vec{r} \cdot \vec{q} \top} \\
& \times \int_{0}^{\infty} \mathrm{d} \ell\left\{\frac{11}{4} v^{4}-2 v_{\top}^{2} v^{2}-b_{z}^{2} \frac{v^{4}}{4 \ell}\right\} \ell^{-(d-1) / 2} e^{-b^{2} / 2 \ell}
\end{aligned}
$$

where we have dropped overall factors, the knowledge of which would require fixing the absolute normalization of the string amplitude. As discussed in the introduction, the first non-vanishing term is of order $O\left(v^{4}\right)$ and its functional behavior in $b$ is independent from $\alpha^{\prime}$.

Notice that, because of the three-body kinematics, the amplitude (2.36) is $O\left(v^{4}\right)$, whereas the interaction of two D-particles without shock wave is $O\left(v^{3}\right)$ 18.

A final comment. The first term on the right-hand side of eq. (2.24) would also give an $\alpha^{\prime}$-independent behavior in $b$, when summed over spin structure and expanded at the order $O\left(v^{2}\right)$. However, since this term is proportional to $(\vec{q} \cdot \vec{v})^{2} / q_{\top}^{2}$, it is sub-leading for $r_{\top} \rightarrow \infty$, and we do not keep it into account in this paper.

\section{The matrix model in the shock wave}

The action in the matrix model is given by the dimensionally reduced $d=10$ and $N=1$ super Yang-Mills action. We are going to compute the one-loop contribution to the effective action in a suitable background.

The gauge-fixed bosonic action is

$$
S_{B}=\int \mathrm{d} t\left[\frac{1}{2} \operatorname{Tr} F_{\mu \nu} F^{\mu \nu}+\operatorname{Tr}\left(D_{\mu}^{B} A^{\mu}\right)^{2}\right]
$$

where

$$
F_{0 i}=\partial_{t} X_{i} \quad \text { and } \quad F_{i j}=i\left[X_{i}, X_{j}\right]
$$

since

$$
A^{i} \equiv X^{i} \quad \text { and } \quad A^{0}=0 .
$$

The action (3.1) is expanded around the classical background field $B_{\mu}$ by separating $A_{\mu}$ into $B_{\mu}$ and the fluctuation $X_{\mu}$ which is integrated out in the path integral. The background covariant derivative is defined by

$$
D_{\mu}^{B} A_{\nu}=\partial_{\mu} A_{\nu}+i\left[B_{\mu}, A_{\nu}\right]
$$

The ghost action is

$$
S_{C}=\int \mathrm{d} t \operatorname{Tr} \bar{C}\left(D^{B}\right)^{2} C,
$$


where the covariant derivative $D$ can be taken equal to $D^{B}$ because we are only interested in the quadratic part of the ghost action.

Similarly, the fermionic action is given by

$$
S_{F}=\int \mathrm{d} t \operatorname{Tr} \bar{\Psi} \Gamma^{\mu} D_{\mu}^{B} \Psi
$$

For the case of two D-particles, the fields

$$
X^{i}=X_{a}^{i} \frac{\tau^{a}}{2}, \quad C=C_{a} \frac{\tau^{a}}{2} \quad \text { and } \quad \Psi=\psi_{a} \frac{\tau^{a}}{2}
$$

take value in the space of the gauge group $S U(2)$ (and $\tau^{a}$ are Pauli's matrices).

In the eikonal approximation, and before introducing the shock wave, the background field $B$ is taken to be

$$
\vec{B}=\left(\begin{array}{cc}
\vec{v}_{1} t+\vec{b}_{1} & 0 \\
0 & \vec{v}_{2} t+\vec{b}_{2}
\end{array}\right) \quad \text { and } \quad B_{0}=0
$$

where $\vec{v}_{i}$ and $\vec{b}_{i}$ are the velocities and positions of the two D-particles. In the frame of reference where $\vec{v}_{1}+\vec{v}_{2}=0$ and $\vec{b}_{1}+\vec{b}_{2}=0$, we can then take

$$
\vec{B}=(\vec{v} t+\vec{b}) \frac{\tau_{3}}{2}
$$

where now $\vec{v}_{1}=-\vec{v}_{2}=\vec{v} / 2, \vec{b}_{1}=-\vec{b}_{2}=\vec{b} / 2 ; v$ and $b$ are the relative velocity and distance in the motion of the two D-particles. As we did for the string case, we have thus factorized out the motion of the center of mass. Notice that the action of the matrix-valued background field on the matrix valued quantum field $\phi$ (where $\phi$ can be $A^{\mu}, C$ or $\left.\psi\right)$ is $B \circ \phi=[B, \phi]$.

\subsection{Kinematics}

The space-time of the shock wave moving along $z$ from right to left is described by the metric element

$$
\mathrm{d} s^{2}=2 \mathrm{~d} U \mathrm{~d} V+h \mathrm{~d} U^{2}+\mathrm{d} \vec{x}_{\top}^{2}
$$

where the light-cone variables $(U, V)$ are defined to be

$$
U \equiv(z+t) / \sqrt{2} \text { and } \quad V \equiv(z-t) / \sqrt{2} \text {. }
$$

The coefficient $h$ is given by

$$
h=f\left(\vec{r}_{\top}\right) \delta(U),
$$

where $\vec{r}_{\top}$ is the distance from the center of mass of the two D-particles to the shock wave. For a source graviton in $d=10$ and with an energy $k_{0}$

$$
f\left(\vec{r}_{\top}\right)=\frac{8 \sqrt{2}}{\pi^{3}} \frac{G_{N} k_{0}}{r_{\top}^{6}},
$$


where $G_{N}$ is Newton's constant.

From now on, we take $U$ as the evolution variable.

The metric components must be thought as a matrix of $S U(2)$ depending on the positions $\vec{Y}_{i}$ of the two D-particles. However,

$$
\left(\begin{array}{cc}
f\left(\vec{Y}_{1}\right) \delta(U) & 0 \\
0 & f\left(\vec{Y}_{2}\right) \delta(U)
\end{array}\right)=\left(\begin{array}{cc}
f\left(\vec{r}_{\top}\right) \delta(U) & 0 \\
0 & f\left(\vec{r}_{\top}\right) \delta(U)
\end{array}\right)\left[1+O\left(\frac{b_{z}}{r_{\top}}\right)\right]
$$

and, for $b_{z} \ll r_{\top}$, we can neglect the higher order terms and take the metric to be proportional to the identity matrix.

Because of the non-flat external metric, care must be taken in lowering and rising indices. In particular, in going from the background gauge fields with lower indices to the background coordinates with upper indices, we must use

$$
B_{U}=h B^{U}+B^{V} \text { and } \quad B_{V}=B^{U}
$$

while $\vec{B}_{\top}=\vec{B}^{\top}$.

We now fix $B^{U}, B^{V}$ and $\vec{B}^{\top}$, by taking into account the trajectories of the Dparticles.

The trajectory of a particle moving in the shock-wave background is (see the appendix)

$$
\left\{\begin{aligned}
V & =U w+w_{0}-\frac{1}{2} f \theta(U) \\
\vec{x}_{\top} & =\vec{v}_{w} U+\vec{b}_{w}
\end{aligned}\right.
$$

By substituting $U=(z+t) / \sqrt{2}$ in (3.16), we reproduce the D-particle trajectories (for $i=1,2$ ) before the shock $z^{(i)}=v_{z}^{(i)} t+b_{z}^{(i)}$ and $\vec{x}_{\top}^{(i)}=\vec{v}_{\top}^{(i)} t+\vec{b}_{\top}^{(i)}$, by the assignment

$$
w^{(i)}=\frac{v_{z}^{(i)}-1}{v_{z}^{(i)}+1}, \quad w_{0}^{(i)}=\frac{\sqrt{2} b_{z}^{(i)}}{v_{z}^{(i)}+1},
$$

and

$$
\vec{v}_{w}^{(i)}=\vec{v}_{\top}^{(i)} \frac{\sqrt{2}}{v_{z}^{(i)}+1}, \quad \vec{b}_{w}^{(i)}=\vec{b}_{\top}^{(i)}-\vec{v}_{\top}^{(i)} \frac{b_{z}^{(i)}}{v_{z}^{(i)}+1} .
$$

The matrix-valued $\vec{B}_{\top}=\left(\vec{x}_{\top}^{(1)}-\vec{x}_{\top}^{(2)}\right) \tau_{3} / 2$ is thus found to be

$$
\vec{B}_{\top}=\left(\frac{\sqrt{2} \vec{v}_{\top}}{1-v_{z}^{2} / 4} U+\vec{b}_{\top}+\frac{\vec{v}_{\top} b_{z} v_{z}}{4-v_{z}^{2}}\right) \frac{\tau_{3}}{2} .
$$

The part of the background proportional to the identity is irrelevant for our computation concerning the relative motion of the D-particles.

Next, we have

$$
\frac{B_{(i)}^{U}+B_{(i)}^{V}}{\sqrt{2}}=z_{(i)}=\frac{U+V_{(i)}}{\sqrt{2}}
$$

and, since $B_{0}=0$ implying $B_{U}=B_{V}$, we impose

$$
h B_{(i)}^{U}+B_{(i)}^{V}=B_{(i)}^{U} .
$$


By solving these constraints at the leading order in $h$, we get the matrix-valued

$$
B_{U}=B_{V}=\frac{v_{z} U+b_{z} / \sqrt{2}}{1-v_{z}^{2} / 4}\left(1+\frac{h}{2}\right) \frac{\tau_{3}}{2} .
$$

Notice the absence of the shift in the trajectory, that is present in the one particle problem; it cancels out in the two-body relative motion. Also, we will see that we only need the leading first-order terms in the velocity in (3.19) and (3.22).

Derivatives in the curved background are often complicated. However, an important property of the shock-wave metric is that, since $\partial_{V} g^{V \lambda}=\partial_{V} \delta(U)=0$, we have that

$$
D_{\nu}^{B} g^{\nu \lambda}=g^{\nu \lambda} D_{\nu}^{B}
$$

with great simplifications in the computation. Moreover, $\sqrt{g}=1$ and the covariant derivative is the usual one. The derivative

$$
\partial_{U}=-\partial_{V}
$$

because the fields do not depend on $z$.

Similarly, for the fermions we can pass the covariant derivative $D_{\mu}^{B}$ through the $\Gamma$-matrices, and use

$$
\operatorname{Tr}_{\gamma} \ln \left(\Gamma_{\mu} D^{\mu}\right)=\frac{1}{2} \operatorname{Tr}_{\gamma} \ln \left(\Gamma_{\mu} D^{\mu}\right)^{2}=\frac{1}{2} \operatorname{Tr}_{\gamma} \ln \left[D^{2}+\frac{1}{2} \Sigma_{\mu \nu} F^{\mu \nu}\right]
$$

even in the shock-wave space-time (the spin connection is zero). Eq. (3.25) simplifies the evaluation of the fermionic path integral.

\subsection{The quadratic action}

Because we are interested in a one-loop computation, we need only the part of the action that is quadratic in the fields. The bosonic action is then

$$
S_{B}=\int \mathrm{d} U \operatorname{Tr} X^{\mu}\left[-\delta_{\mu}^{\nu} D^{2}-2 i F_{\mu}^{\nu}\right] X_{\nu}
$$

In eq. (3.26), the background field strength $F_{\nu}^{\mu}$ is independent from $U$. As we shall see, because the result is already proportional to $F^{4}$, we only need the part of $F$ that is leading order in $v$ and which is given by $(\mu, \nu=U, V, \top)$

$$
F_{\mu}^{\nu}=\left(\begin{array}{ccc}
2 v_{z} & 0 & \sqrt{2} v_{\top} \\
0 & -2 v_{z} & -\sqrt{2} v_{\top} \\
\sqrt{2} v_{\top} & -\sqrt{2} v_{\top} & 0
\end{array}\right)+h\left(\begin{array}{ccc}
v_{z} & -2 v_{z} & 0 \\
0 & -v_{z} & 0 \\
0 & -\sqrt{2} v_{\top} & 0
\end{array}\right) .
$$

Notice that in writing (3.27), we used $\partial_{V} h=0$ as well as $\partial_{U} h \simeq 0$ (consistently with (3.24) ) since terms proportional to $\delta^{\prime}(U)$ would eventually multiply functions of $U^{2}$ in the final amplitude and would not contribute. 
In order to compute the amplitude for the scattering of the two D-particles in the external field of the shock wave, we need

$$
\begin{aligned}
a\left(r_{\top}, b, v\right)= & \ln \int[\mathrm{d} X][\mathrm{d} \bar{C}][\mathrm{d} C][\mathrm{d} \Psi][\mathrm{d} \bar{\Psi}] e^{-S_{B}-S_{C}-S_{F}}=-\frac{1}{2} \operatorname{Tr} \ln \left(-\delta_{\mu}^{\nu} D^{2}-2 i F_{\mu}^{\nu}\right) \\
& +\operatorname{Tr} \ln \left(-D^{2}\right)+\frac{1}{4} \operatorname{Tr} \ln \left(-D^{2}-\frac{1}{2} \Sigma_{\mu \nu} F^{\mu \nu}\right) .
\end{aligned}
$$

The operator traces can be written in terms of the Schwinger representation by introducing a parameter $s$. The amplitude (3.28) thus becomes

$$
a\left(r_{\top}, b, v\right)=a_{B}+a_{F},
$$

where

$$
a_{B}=-\frac{1}{2} \int \mathrm{d} U \int_{0}^{\infty} \frac{\mathrm{d} s}{s} \lim _{U_{1,2} \rightarrow U} \mathcal{W}_{g}\left(s, U_{1}, U_{2}\right)\left(\operatorname{Tr}_{\mu \nu} e^{2 i s F_{\mu}^{\nu}}-2\right)
$$

for the bosonic part, and

$$
a_{F}=+\frac{1}{4} \int \mathrm{d} U \int_{0}^{\infty} \frac{\mathrm{d} s}{s} \lim _{U_{1,2} \rightarrow U} \mathcal{W}_{g}\left(s, U_{1}, U_{2}\right)\left(\operatorname{Tr}_{\gamma} e^{s \Sigma_{\mu \nu} F^{\mu \nu} / 2}\right)
$$

for the fermionic one.

In (3.30) and (3.31) we have separated between parenthesis the part that does depend on $U$ and defined

$$
\mathcal{W}_{g}\left(s, U_{1}, U_{2}\right)=\left\langle U_{1}\left|e^{s D^{2}}\right| U_{2}\right\rangle
$$

the kernel of the scalar propagator in the shock wave space-time, where, to leading order in $h, D^{2}$ is given by

$$
\begin{aligned}
D^{2} & =2 \partial_{U} \partial_{V}-2 B_{U} B_{V}-\vec{B}_{\top} \cdot \vec{B}_{\top}+h B_{V} B_{V}-h \partial_{V}^{2} \\
& =-2 \partial_{U}^{2}-2 v^{2} U^{2}-b^{2}+h\left[-\partial_{U}^{2}-\frac{b_{z}^{2}}{2}\right] .
\end{aligned}
$$

In writing (3.33), we have used (3.19), (3.22) and (3.24) after replacing for $V$ and $x_{\top}$ the values on the trajectory. In the the term proportional to $h$, we have neglected contributions proportional to $v$ because this part is going to be multiply by $v^{4}$. The operator $D^{2}$ acts on the components $\phi_{a}$ of the matrix-valued field $\phi=\phi^{a} \tau_{a} / 2$ and $B_{U}$, $B_{V}$ and $B_{\top}$ in eq. (3.33) are the coefficients in front of $\tau_{3} / 2$ in eq. (3.22) that remain after performing the trace over the gauge group. Terms linear in $B_{\mu}$ do not contribute to the trace since $B \propto \tau_{3}$.

The kernel $(\underline{3.32})$ can be expanded around the flat space-time $(h=0)$ part:

$$
\mathcal{W}_{g}(s, U) \equiv \lim _{U_{1}, U_{2} \rightarrow U} \mathcal{W}_{g}\left(s, U_{1}, U_{2}\right)=\mathcal{W}_{\eta}(s, U)+h \Omega(s, U)+O\left(h^{2}\right)
$$

where

$$
\mathcal{W}_{\eta}(s, U)=\lim _{U_{1,2} \rightarrow U}\left\langle U_{1}\left|e^{-s\left(\partial_{U}^{2}+2 v^{2} U^{2}+b^{2}\right)}\right| U_{2}\right\rangle
$$


is just the kernel for the harmonic oscillator, that is

$$
\mathcal{W}_{\eta}(s, U)=\sqrt{\frac{v}{2 \pi \sin 4 v s}} e^{-s b^{2}} e^{v U^{2}(\cos 4 v s-1) / \sin 4 v s} .
$$

For the flat space-time case, (3.28) reproduces the known result [18, 3, 5] for the phase shift of two interacting D-particles. In fact, traces of odd powers of $F_{\nu}^{\mu}$ vanish and we have that

$$
\operatorname{Tr}_{\mu \nu} e^{2 i s F_{\mu}^{\nu}}-2=(10-2)+2(\cos 4 v s-1)
$$

and

$$
\operatorname{Tr}_{\gamma} e^{s \Sigma_{\mu \nu} F^{\mu \nu} / 2}=16 \cos 2 v s
$$

since $\operatorname{Tr}_{\mu \nu} F^{2}=8 v^{2}$ and

$$
\operatorname{Tr}_{\gamma}\left(\Sigma_{\mu \nu} F^{\mu \nu}\right)^{2}=-(16 \times 2) \operatorname{Tr}_{\mu \nu} F^{2}
$$

where the factor 16 comes from the Dirac trace.

By taking the traces in (3.30) and (3.31) we thus find

$$
a(b, v) \simeq \int_{0}^{\infty} \frac{\mathrm{d} s}{s} \int \mathrm{d} U \mathcal{W}_{\eta}(s, U)[4 \cos 2 v s-\cos 4 v s-3]
$$

where

$$
\int \mathrm{d} U \mathcal{W}_{\eta}(s, U)=\frac{e^{-s b^{2}}}{2 \sin 2 s v} .
$$

In the light-cone formalism, we reproduce the formulas of refs. [18, 3, 5] with $2 v$ in the place of $v$ there. The different factor in front of $v$ is absorbed in the overall normalization since the use of these formulas for string theory makes sense only up to $O\left(v^{4}\right)$ (actually, in the flat case, $O\left(v^{3}\right)$, due to eq. (3.41)).

As we shall see, contrary to the flat space-time case, the amplitude in the shockwave background is proportional to $h=f\left(r_{\top}\right) \delta(U)$, and therefore the integration over $U$ yields $\int \mathrm{d} U h \mathcal{W} \simeq f\left(r_{\top}\right) e^{-s b^{2}}$ instead of (3.41). In agreement with the string amplitude (2.36), the amplitude in the curved background is thus $O\left(v^{4}\right)$ rather than $O\left(v^{3}\right)$, as in the flat case.

\subsection{The scattering amplitude}

In order to compute (3.28) in the non-flat metric, we must expand the exponential functions in (3.30) and (3.31) in powers of $h$. To the leading order in $h$, we have that

$$
\operatorname{Tr}_{\mu \nu} F^{2}=8\left(v^{2}+h v_{z}^{2}\right), \quad \operatorname{Tr}_{\mu \nu} F^{4}=32\left(v^{4}+2 h v_{z}^{2} v^{2}\right) .
$$

As in the flat-space case above, (3.39) holds together with

$$
\operatorname{Tr}_{\gamma}\left(\Sigma_{\mu \nu} F^{\mu \nu}\right)^{4}=-(16 \times 16) \operatorname{Tr}_{\mu \nu} F^{4}
$$


and it is true in general that $\left(\operatorname{Tr} F^{2}\right)^{2}=2 \operatorname{Tr} F^{4}$.

We can thus expand to fourth order the exponential functions and write that

$$
-\frac{1}{2}\left(\operatorname{Tr}_{\mu \nu} e^{2 i s F_{\mu}^{\nu}}-2\right)+\frac{1}{4}\left(\operatorname{Tr}_{\gamma} e^{s \Sigma_{\mu \nu} F^{\mu \nu} / 2}\right)=-\frac{s^{4}}{4} \operatorname{Tr} F^{4}
$$

where the constant terms as well as those quadratic in the velocity have cancelled as it happens in the flat-metric case.

By means of (3.44), we can now write (3.28) as

$$
a\left(r_{\top}, b, v\right) \simeq \int \mathrm{d} U \int \frac{\mathrm{d} s}{s}\left[\mathcal{W}_{\eta}(s, U)+h \Omega(s, U)\right]\left(-\frac{s^{4}}{4} \operatorname{Tr} F^{4}\right),
$$

where $\Omega(s, U)$ is given by

$$
\begin{aligned}
\Omega(s, U) & =-\lim _{U_{1,2} \rightarrow U} s\left[\partial_{U_{1}}^{2}+\frac{b_{z}^{2}}{2}\right] \mathcal{W}_{\eta}\left(s, U_{1}, U_{2}\right) \\
& =-s\left[\frac{1}{4 s}+\frac{b_{z}^{2}}{2}\right] \mathcal{W}_{\eta}(s, U),
\end{aligned}
$$

and $\mathcal{W}_{\eta}$ by (3.36).

Collecting all terms linear in $h$ yields, apart for an overall factor:

$$
a\left(r_{\top}, b, v\right) \simeq 4 \int \mathrm{d} U f\left(\vec{r}_{\top}\right) \delta(U) \int \mathrm{d} s s^{5 / 2} e^{-s b^{2}}\left\{\frac{7}{4} v^{4}-2 v^{2} v_{\top}^{2}-s b_{z}^{2} \frac{v^{4}}{2}\right\}
$$

where we have now taken (3.36) at $v=0$.

\subsection{Comparison with the string result}

Going back to the string computation, the amplitude (2.36) is what we want to compare with the matrix theory result in (3.47). After changing $\ell \rightarrow 1 / 2 s$, setting $d=10$, and normalizing the incoming and outgoing source-graviton states each by the usual factor $1 / \sqrt{k_{0}}$, we obtain that the string amplitude is (up to an overall constant)

$$
a\left(r_{\top}, b, v\right) \simeq f\left(\vec{r}_{\top}\right) \int \mathrm{d} s s^{5 / 2} e^{-s b^{2}}\left\{\frac{11}{4} v^{4}-2 v_{\top}^{2} v^{2}-s b_{z}^{2} \frac{v^{4}}{2}\right\} .
$$

since

$$
f\left(\vec{r}_{\top}\right) \propto k_{0} \int \frac{\mathrm{d}^{8} \vec{q}_{\top}}{(2 \pi)^{8}} \frac{1}{\vec{q}_{\top}^{2}} e^{i \vec{r}_{\top} \cdot \vec{q} \uparrow}
$$

After integrating (3.47) over $U$, we see that the matrix model result (up to an overall constant) becomes equal to that of string theory except for the numerical factor $7 / 4$ instead of $11 / 4$ in front of the $v^{4}$ term. This missing term comes from the inclusion of the Jacobian arising from the $\delta$-function constraining the D-particles to lie on their respective trajectories, which in our formalism amounts to implementing (3.20) and 
(3.21) for both. In order to enforce this constraint, we replace the integration over $U$ by

$$
\begin{aligned}
\int \mathrm{d} U \rightarrow & \int \mathrm{d} U \prod_{i=1,2} \int \mathrm{d} B_{(i)}^{U} \mathrm{~d} B_{(i)}^{V} \delta\left(B_{(i)}^{U}+B_{(i)}^{V}-\sqrt{2} z^{(i)}\right) \delta\left(B_{(i)}^{U}(1-h)-B_{(i)}^{V}\right) \\
& \simeq \frac{1}{4} \int \mathrm{d} U(1+h) \\
& \times \prod_{i=1,2} \int \mathrm{d} B_{(i)}^{U} \mathrm{~d} B_{(i)}^{V} \delta\left(B_{(i)}^{U}-\frac{1}{\sqrt{2}}\left(1+\frac{h}{2}\right) z^{(i)}\right) \delta\left(B_{(i)}^{V}-\frac{1}{\sqrt{2}}\left(1-\frac{h}{2}\right) z^{(i)}\right) .
\end{aligned}
$$

The extra (leading in $h$ ) factor $1+h$ in front, after multiplication by $-s^{4} \operatorname{Tr} F^{4} / 4$, provides the missing term $4 s^{4} v^{4} h$ that adds a term $v^{4}$ to (3.48) and makes the matrixmodel result agree with that of string theory, since $7 / 4+1=11 / 4$, and we finally obtain that

$$
a\left(r_{\top}, b, v\right) \simeq f\left(\vec{r}_{\top}\right) \frac{v^{2}}{b^{7}}\left[\frac{11}{4} v^{2}-2 v_{\top}^{2}-\frac{7}{4} \frac{b_{z}^{2}}{b^{2}} v^{2}\right] .
$$

\section{Acknowledgments}

R. I. would like to acknowledge the collaboration of C. Núñez and F. Hussain in the early stages of this work concerning the string computation, and E. Gava, F. Morales and K. S. Narain for useful discussions. All three authors thank G. Ferretti for many discussions.

\section{A. Notation and useful formulas}

We collect in this appendix formulas and results that we used in the previous sections but were too cumbersome to be inserted in the main text.

\section{A.1. String propagators}

The bosonic propagator is given by (see, for instance, [19])

$$
\left\langle X^{\mu}(z) X^{\nu}(w)\right\rangle=-\eta^{\mu \nu} G(z, w)-S^{\mu \nu} G(z, \bar{w}),
$$

where the metric signature is given by $\eta^{\mu \nu}=\left(-1, \delta^{i j}\right)$,

$$
G(z, w)=\frac{1}{4 \pi} \ln \left|\frac{\vartheta_{1}(z-w)}{\vartheta_{1}^{\prime}(0)}\right|-\frac{\operatorname{Im}^{2}(z-w)}{2 \operatorname{Im} \tau},
$$

$S^{00}=-1$ and $S^{i j}=-\delta^{i j}$. Everywhere, we write

$$
\vartheta_{i}(x) \equiv \vartheta_{i}(x \mid \tau)
$$

where $\tau=2 i \ell$. 
The fermionic propagator at velocity $v=0$ is

$$
\left\langle\psi^{\mu}(z) \psi^{\nu}(w)\right\rangle_{S}=-\frac{\eta^{\mu \nu}}{4 \pi} P_{S}
$$

where

$$
P_{S}=\frac{\vartheta_{S}(z-w) \vartheta_{1}^{\prime}(0)}{\vartheta_{1}(z-w) \vartheta_{S}(0)}
$$

At $v \neq 0$ we have that (here the direction 1 is parallel and the directions $i, j$ are orthogonal to $\vec{v}$ ) 20

$$
\begin{aligned}
\left\langle\psi^{0}(z) \psi^{0}(w)\right\rangle_{S} & =\left\langle\psi^{1}(z) \psi^{1}(w)\right\rangle_{S}=\frac{1}{4 \pi} Q_{S} \\
\left\langle\psi^{0}(z) \psi^{1}(w)\right\rangle_{S} & =\left\langle\psi^{1}(z) \psi^{0}(w)\right\rangle_{S}=\frac{1}{4 \pi} R_{S} \\
\left\langle\psi^{i}(z) \psi^{j}(w)\right\rangle_{S} & =\frac{\delta^{i j}}{4 \pi} P_{S},
\end{aligned}
$$

where the spin-structure-dependent functions are defined as

$$
\begin{aligned}
& Q_{S}=\frac{1}{2}\left[e^{v} \frac{\vartheta_{S}(w-i v / \pi) \vartheta_{1}^{\prime}(0)}{\vartheta_{S}(-i v / \pi) \vartheta_{1}(w)}+e^{-v} \frac{\vartheta_{S}(w+i v / \pi) \vartheta_{1}^{\prime}(0)}{\vartheta_{S}(-i v / \pi) \vartheta_{1}(w)}\right] \\
& R_{S}=\frac{1}{2}\left[e^{v} \frac{\vartheta_{S}(w-i v / \pi) \vartheta_{1}^{\prime}(0)}{\vartheta_{S}(-i v / \pi) \vartheta_{1}(w)}-e^{-v} \frac{\vartheta_{S}(w+i v / \pi) \vartheta_{1}^{\prime}(0)}{\vartheta_{S}(-i v / \pi) \vartheta_{1}(w)}\right]
\end{aligned}
$$

In performing the sum over the spin structure, it useful to use Riemann's identity:

$$
\frac{1}{2} \sum_{S}( \pm)_{S} \vartheta_{S}(u) \vartheta_{S}(v) \vartheta_{S}(w) \vartheta_{S}(s)=\vartheta_{1}\left(u_{1}\right) \vartheta_{1}\left(v_{1}\right) \vartheta_{1}\left(w_{1}\right) \vartheta_{1}\left(s_{1}\right)
$$

where

$$
\begin{aligned}
u_{1}=(u+v+w+s) / 2 & v_{1}=(u+v-w-s) / 2 \\
w_{1}=(u-v+w-s) / 2 & s_{1}=(u-v-w+s) / 2 .
\end{aligned}
$$

\section{A.2. Shock-wave metric}

The space-time of the shock wave is defined by the metric element (3.10) which gives a metric tensor (its signature is such that $\eta_{00}=-1$ ) with components:

$$
g_{U U}=h, \quad g_{U V}=1, \quad g_{V V}=0, \quad g_{i j}=\delta_{i j}
$$

and

$$
g^{U U}=0, \quad g^{U V}=1, \quad g^{V V}=-h, \quad g^{i j}=\delta^{i j},
$$

where $h=f\left(r_{\top}\right) \delta(U)$.

Similarly, the einbein, necessary in writing the fermionic action, is given by

$$
e_{U}^{U}=e_{U}^{V}=1, \quad e_{V}^{U}=0, \quad e_{U}^{V}=\frac{h}{2}, \quad e_{i}^{j}=\delta_{j}^{i}
$$


where $e_{\mu}^{a} \eta_{a b} e_{\nu}^{b}=g_{\mu \nu}$. The spin connection is zero: $\omega_{a b \mu}=0$.

The action for the motion of one particle in the shock-wave metric is found by varying the metric line element (3.10) and it is given by

$$
S=\int \mathrm{d} s\left[2 \frac{\mathrm{d} U}{\mathrm{~d} s} \frac{\mathrm{d} V}{\mathrm{~d} s}+f \delta(U)\left(\frac{\mathrm{d} U}{\mathrm{~d} s}\right)^{2}+\left(\frac{\mathrm{d} \vec{x}_{\top}}{\mathrm{d} s}\right)^{2}\right] .
$$

The trajectory is therefore

$$
\left\{\begin{aligned}
V & =U w+w_{0}-\frac{1}{2} f \theta(U) \\
U & =s \\
\vec{x}_{\top} & =\vec{v}_{w} U+\vec{b}_{w},
\end{aligned}\right.
$$

where the coefficients are given in section 3 by eqs. (3.17) and (3.18). 


\section{References}

[1] J. Polchinski, String Theory (Cambridge University Press, Cambridge, 1998).

[2] U. H. Danielsson, G. Ferretti and B. Sundborg, Int. J. Mod. Phys. A 11 (1996) 5463, hep-th/9603081;

D. Kabat and P. Pouliot, Phys. Rev. Lett. 77 (1996) 1004, hep-th/9603127.

[3] M. R. Douglas, D. Kabat, P. Pouliot and S. H. Shenker, Nucl. Phys. B 485 (1997) 85, hep-th/9608024.

[4] T. Banks, W. Fischler, S. H. Shenker and L. Susskind, Phys. Rev. D 55 (1997) 5112 hep-th/9610043;

L. Susskind, hep-th/9704080.

[5] K. Becker and M. Becker, Nucl. Phys. B 506 (1997) 48 hep-th/9705091;

K. Becker, M. Becker, J. Polchinski and A. Tseytlin, Phys. Rev. D 56 (1997) 3174 hep-th/9706072.

[6] M. Fabbrichesi, G. Ferretti and R. Iengo, JHEP 06 (1998) 002 hep-th/9806018;

[7] Y. Okawa and T. Yoneya, hep-th/9806108 and hep-th/9808188.

[8] M. Dine and A. Rajaraman, hep-th/9710174;

M. Dine, R. Echols and J.P. Gray, hep-th/9805007;

W. Taylor IV and M. Van Raamsdonk, hep-th/9806066;

R. Echols and J.P. Gray, hep-th/9806109;

M. Fabbrichesi, G. Ferretti and R. Iengo, hep-th/9806166.

[9] M. R. Douglas, hep-th/9707228;

I. Ya. Aref'eva and I. V. Volovich, hep-th/9802091.

[10] G. T. Horowitz and E. J. Martinesc, Phys. Rev. D 57 (1998) 4935 [hep-th/9710217];

T. Banks, W. Fischler, I. R. Klebanov and L. Susskind, JHEP 01 (1998) 008 hep-th/9711005;

K. Becker and M. Becker, hep-th/9810050.

[11] G. 't Hooft, Phys. Lett. B 198 (1987) 61.

[12] D. Amati and C. Klimcik, Phys. Lett. B 219 (1989) 443.

[13] G. T. Horowitz and A. R. Steif, Phys. Rev. Lett. 64 (1990) 260

[14] M. Fabbrichesi and K. Roland, Nucl. Phys. B 388 (1992) 539.

[15] M. Fabbrichesi, R. Iengo and K. Roland, Nucl. Phys. B 402 (1993) 360.

[16] G. T. Horowitz and N. Itzhaki, hep-th/9901012.

[17] A. Pasquinucci, hep-th/9703066.

[18] C. Bachas, Phys. Lett. B 374 (1996) 37, hep-th/9511043.

[19] F. Hussain, R. Iengo and C. Núñez, Nucl. Phys. B 497 (1997) 205.

[20] F. Hussain, R. Iengo, C. Núñez and C. A. Scrucca, Nucl. Phys. B 517 (1998) 92. 